\title{
Klassifizierung der Methoden eines Ganzheitlichen Produktionssystems unter Berücksichtigung der Anforderungen indirekter Bereiche
}

\author{
Classification of Methods of a Comprehensive Production System, \\ Taking into Account the Requirements of Overhead Areas
}

\author{
Sebastian Fehr \\ Kay Sauber \\ Thorsten Schmidt \\ Professur für Technische Logistik \\ Technische Universität Dresden
}

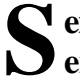
eit den 50er Jahren des Zwanzigsten Jahrhunderts erfährt das Konzept der schlanken Produktion stetig Erweiterungen und Renaissancen [Fau09]. Zielt das Ursprungskonzept noch überwiegend auf eine Effizienzsteigerung im Bereich der Fertigung ab, so werden im Rahmen eines Ganzheitlichen Produktionssystems verstärkt auch die indirekten Bereiche, wie z. B. Konstruktion, Forschung und Entwicklung, Planung oder Vertrieb einbezogen. Im folgenden Beitrag werden die Rahmenbedingungen eines Produktionssystems im indirekten Bereich dargestellt und Empfehlungen hinsichtlich einer strukturellen Erweiterung aktuell bestehender Ordnungsrahmen gegeben.
\end{abstract}

[Schlüsselwörter: Lean Management; schlanke Produktion; Ganzheitliches Produktionssystem; Indirekter Bereich; kontinuierlicher Verbesserungsprozess]

Sin ince the 1950s, the concept of lean production has experienced steady expansion and the concept has been revived after a period of dwindling use [Fau09]. Aims of the original concept are predominantly to increase efficiency in manufacturing, so within the context of a comprehensive production system, overhead areas including design, research and development, planning or sales are also strengthened. In the following article, frameworks of a production system in overhead areas are shown and recommendations are made regarding a structural extension of the currently existing regulatory framework.

[Keywords: lean management; lean production; comprehensive production system; indirect divisions; continuous improvement process]

\section{EINFÜHRUNG}

\subsection{RAHMENBEDINGUNGEN}

Unternehmen sehen sich zunehmend mit immer radikaleren Veränderungen innerhalb kürzer werdenden Intervallen konfrontiert [vgl. Mos09]. Neben der fortschreitenden Globalisierung der Beschaffungs- und Absatzmärkte sowie der Zunahme der technischen Produktkomplexität ist insbesondere eine immer differenziertere Segmentierung der Kundengruppen $\mathrm{zu}$ beobachten [vgl. Kra03]. Die Unternehmen reagieren häufig mit einer wachsenden Anzahl an Derivaten sowie der Verkürzung des Time-to-Market. Durch die steigende Vielfalt der Leistungsvarianten verschiebt sich der Grad der Leistungswiederholung tendenziell zu kleineren Stückzahlen [Mil00]. Die Komplexität und erforderliche Variabilität des Wertschöpfungssystems steigt [Spe00]. Um den kontinuierlich wachsenden Anforderungen gerecht zu werden und sich unter den genannten Rahmenbedingungen nachhaltig am Markt entwickeln zu können, wird es auch im Hinblick auf begrenzte Ressourcen immer wichtiger, die Flexibilität, Produktivität und Effizienz der Leistungserstellung signifikant und fortwährend zu verbessern. Zur Erreichung dieser strategischen Unternehmensziele gelten Ganzheitliche Produktionssysteme sowohl im theoretischen als auch betrieblichen Diskurs als probates Mittel [vgl. Bau02; Spa03].

\subsection{Ganzheitliche Produktionssysteme}

Ganzheitliche Produktionssysteme verfolgen das Ziel bereits vorhandene Konzepte und Modernisierungsmaßnahmen, welche meist zur Verbesserung eines konkreten Sachverhalts eingeführt wurden, im Rahmen eines ganzheitlichen Ansatzes zu einem modularen, methodisch aufeinander abgestimmten System zu vereinen [Lay08]. Dabei werden bekannte Methoden sowohl zueinander in Bezug gesetzt als auch mit der Unternehmensstrategie 
verknüpft. So soll vermieden werden, dass mit einer isoliert betrachteten Vorgehensweise jeweils nur optimale Teillösungen realisiert werden, die unter Umständen zu Verschlechterungen in einem anderen Bereich führen [Lay08a]. Grundsätzlich kann davon ausgegangen werden, dass die Struktur Ganzheitlicher Produktionssysteme gleich ist, die Inhalte sich jedoch nach dem jeweiligen unternehmensspezifischen Kontext differenzieren. In der Regel bestehen sie aus den in der Unternehmensstrategie formulierten Zielen, den Unternehmensprozessen, den Gestaltungsprinzipien sowie den zugeordneten Methoden und Werkzeugen (vgl. Abbildung 1).

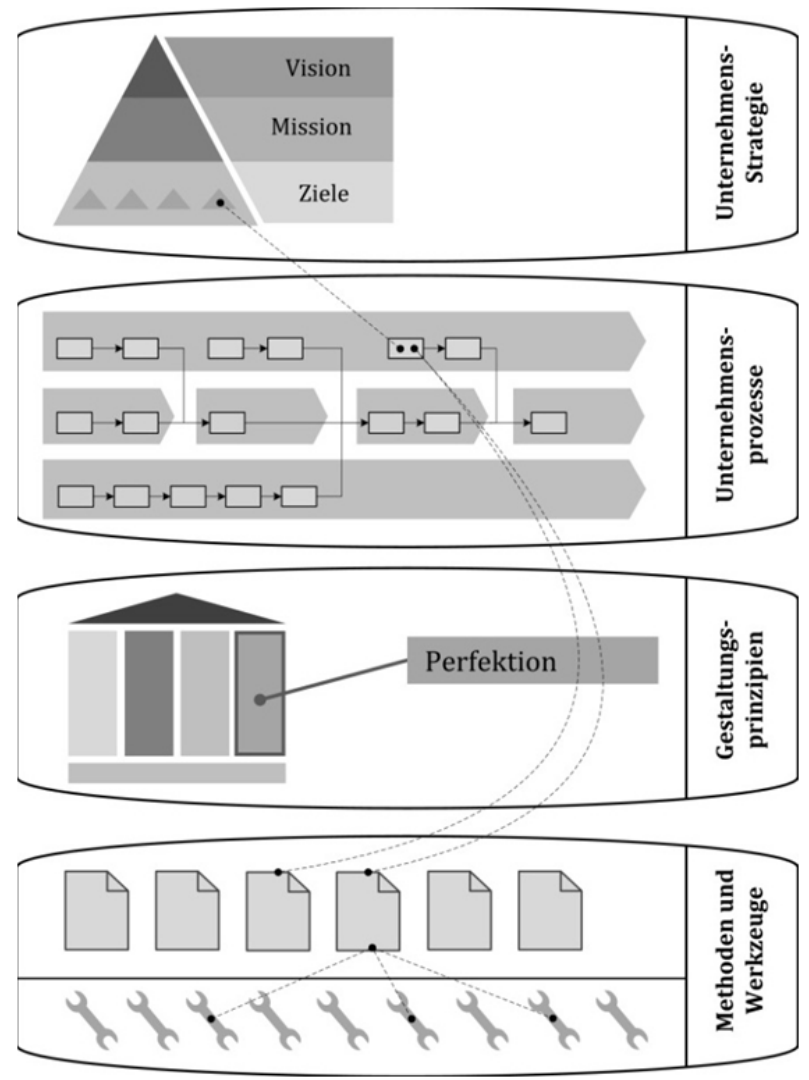

Abbildung 1. Aufbau eines GPS [vgl. VDI2870]

Ziel eines Ganzheitlichen Produktionssystems ist es, in einem kontinuierlichen, vom Mitarbeiter getragenen Prozess bestehende Abläufe zu hinterfragen und unter Anwendung der durch einen Ordnungsrahmen strukturierten Methoden fortwährend und im Sinne der Unternehmensstrategie zu verbessern [Fe11]. Für den nachhaltigen Erfolg ist es unabdingbar, dass das Produktionssystem von allen Mitarbeitern auf sämtlichen Ebenen des Unternehmens verstanden, akzeptiert und umgesetzt wird [VDI2870]. Im vorliegenden Beitrag werden die Rahmenbedingungen einer Erweiterung des aus der Fertigung stammenden Managementkonzepts auf die indirekten Unternehmensbereiche beschrieben und ein Ansatz für eine bereichsübergreifende Gestaltung des Ordnungsrahmens zur verbesserten Methodenstrukturierung und -auswahl aufgezeigt.

\subsection{BEGRIFFSKLÄRUNG}

Im Rahmen dieser Veröffentlichung werden unter dem indirekten Bereich alle Organisationseinheiten zusammengefasst, die nicht unmittelbar an der Erstellung von kundenrelevanten Produkten (direkter Bereich) beteiligt sind. Zum indirekten Bereich zählen somit nicht nur die rein administrativen Bereiche, sondern auch produktionsvorbereitende und -begleitende Struktureinheiten mit planenden und gestaltenden Aufgaben.

\section{TREND ZUR INTEGRATION INDIREKTER UNTERNEHMENSBEREICHE}

Ein Aspekt der Ganzheitlichkeit beruht auf dem Anspruch innerhalb eines umfassenden Ordnungsrahmens eine Hilfestellung für sämtliche im Unternehmen ablaufenden Prozesse realisieren zu können [Spa03; Korg04]. Die betriebliche Praxis zeigt, dass Probleme, die im Bereich der Fertigung auftreten, häufig nur unter Einbeziehung indirekter Bereiche gelöst werden können [Fe11]. Um die Potenziale im direkten Bereich in vollem Umpfang nutzen zu können, ist somit ein vertieftes Verständnis des Produktionssystems auch in den indirekten Bereichen obligatorisch. Auch Ohno, der Begründer des Toyota-Produktionssystems, ist davon überzeugt, dass die ganze Stärke seines Systems sich ,erst in der Anwendung als umfassendes Managementsystem (...)“ offenbart [Ohn93]. Darüber hinaus sind es gerade die indirekten Bereiche, denen zukünftig die höchsten Effizienzsteigungspotenziale attestiert werden [Wit06]. So ist es nicht verwunderlich, dass eine wachsende Zahl an Unternehmen sich damit beschäftgt, das Einsatzgebiet ihres Ganzheitlichen Produktionssystems konsequent auf die indirekten Bereiche auszuweiten [Spa04].

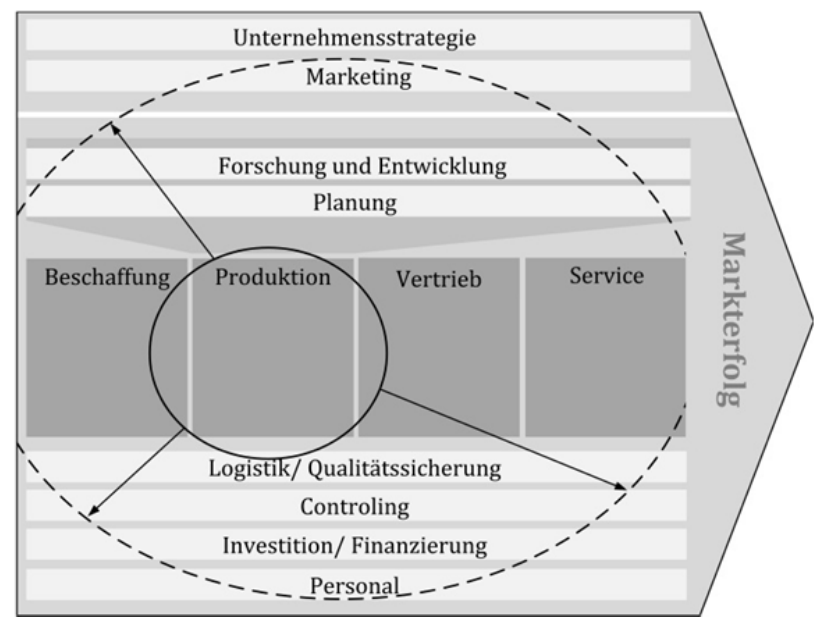

Abbildung 2. Ausweitung des Anwendungsgebietes auf indirekte Bereiche (i. A. a. [Töpf07; Port99; Keß09])

In diesem Zusammenhang wurden häufig zunächst die Ansätze aus der Stückgutproduktion, soweit möglich, auf die indirekten Geschäftsprozesse übertragen. Dabei ist 
festzustellen, dass eine sinnvolle Anwendung dieser Methoden aufgrund unterschiedlicher Rahmenbedingungen nur in gewissen Teilbereichen möglich ist. Um dem Anspruch der Ganzheitlichkeit gerecht zu werden, ist es notwendig, diese unterschiedlichen Rahmenbedingungen zu beachten. Nur wenn die Differenzierungsmerkmale der Bereiche berücksichtigt werden, ist es möglich, einen zielgerichteten und damit effektiven Verbesserungsprozess zu etablieren.

\subsection{RAHMENBEDINGUNGEN UND ANFORDERUNGEN EINES GANZHEITLICHEN PRODUKTIONSSYSTEMS IM INDIREKTEN BEREICH}

Zunächst ist festzustellen, dass die im Rahmen von Produktionssystemen häufig gewählte Differenzierung in direkte und indirekte Bereiche (vgl. Kap. 1.3) für eine Unterscheidung hinsichtlich der Anwendbarkeit von Methoden eines Produktionssystems nur bedingt geeignet ist. Dies liegt im Wesentlichen an der Heterogenität der in den Bereichen zu bewältigenden Aufgaben. So reicht die Vielfalt der Tätigkeiten im indirekten Bereich von regelmäßig zu wiederholenden administrativen Tätigkeiten bis hin zu kreativen Planungs- und Entwicklungsaufgaben mit Projektcharakter. Durch die Übertragung von Methoden aus dem Bereich der Fertigung wird aufgrund ähnlicher Prozessgegebenheiten vorwiegend der administrative Bereich abgedeckt (vgl. Abbildung 3).

\begin{tabular}{|c|c|c|}
\hline Direkter Bereich & \multirow{2}{*}{\multicolumn{2}{|c|}{$\begin{array}{l}\text { überwiegend kreative Tätigkeiten } \\
\text { mit wechselnder Abfolge }\end{array}$}} \\
\hline \multirow[t]{2}{*}{ Fertigung } & & \\
\hline & \multicolumn{2}{|r|}{ Indirekter Bereich } \\
\hline \multirow{7}{*}{$\begin{array}{l}\text { überwiegend repetitive Tätigkeiten } \\
\text { mit klar definierter Abfolge }\end{array}$} & \multirow{7}{*}{ 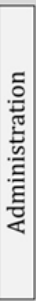 } & Management \\
\hline & & Forschung und Entwicklung \\
\hline & & Planung \\
\hline & & Logistik/ Qualitätssicherung \\
\hline & & Investition/ Finanzierung \\
\hline & & Controling \\
\hline & & Personal \\
\hline
\end{tabular}

\section{Abbildung 3. Differenzierte Rahmenbedingungen}

Auch die dort ausgeübten Prozesse weisen einen hohen Standardisierungsgrad sowie eine hohe Wiederholungsrate auf. Darüber hinaus sind die zu erreichenden Ziele bekannt und ausführlich beschreibbar. Das wesentlichste Unterscheidungsmerkmal liegt in der Stofflichkeit des Leistungsergebnisses. Während im direkten Bereich physische Produkte erstellt werden, ist das Ergebnis im administrativen Bereich in der Regel eine Dienstleistung. Neben den repetitiven, administrativen Tätigkeiten existieren in den indirekten Bereichen jedoch ebenso Bereiche, die einer hohen Dynamik ausgesetzt sind. So sind Ziele in einem Planungsprozess, aufgrund des Anspruchs möglichst schnell auf wechselnde Umweltbedingungen reagieren zu können, häufig zunächst diffus und konkretisieren sich erst während des Projektverlaufs. Auch die Wiederholungsrate der Prozesse ist deutlich geringer. Darüber hinaus sind die Problemstellungen in der Regel bereichsübergreifend, sodass der Ort an dem ein Problem relevant wird, häufig nicht mit dem Ort der Problementstehung übereinstimmt. Somit reicht es insbesondere im indirekten Bereich nicht aus, den Mitarbeitern die Aufgabe der kontinuierlichen Verbesserung $\mathrm{zu}$ übertragen. Vielmehr muss der Impuls für den Verbesserungsprozess aus dem Management kommen und nach Kräften unterstützt werden [Fe11].

Zusammenfassend ist festzustellen, dass durch die reine Anwendung der aus dem Bereich der Fertigung stammenden Methoden nur ein Bruchteil der im indirekten Bereich auftretenden Problemstellungen systematisch einer Lösung zugeführt werden kann. Somit wird es notwendig im Rahmen eines ganzheitlichen Ansatzes weitere Methoden (z. B. Prozessmapping, Problemlösung) einzubeziehen. Ist es im Bereich der Fertigung und Montage sowie bei administrativen Tätigkeiten aufgrund der immer wiederkehrenden und an vielen Stellen des Unternehmens in gleicher Weise ablaufenden Prozesse möglich, durch spezifische Lösungsansätze Verbesserungen zu erzielen, so sind die Problemstellungen bspw. im Bereich der Planung individuellerer Natur. Im ersten Fall ist es möglich, an diversen Stellen im Unternehmen durch Experten vorgefertigte Lösungen zu implementieren, die zu einer unmittelbaren Effizienzsteigerung führen. Im Bereich des Engineerings sitzen die zur Problemlösung benötigten Experten aufgrund der Vielfältigkeit der Problemstellungen meist dezentral in den jeweiligen Organisationseinheiten. Hier ist es notwendig, durch eine strukturierte Herangehensweise, die Bereiche methodisch bei der Lokalisierung und Eliminierung von Problemen zu unterstützen. Dies ist auch der Grund dafür, dass die Methoden im Bereich der Fertigung bzw. Administration bereits definierte Lösungen für konkrete Problemstellungen bieten. Im Bereich des Engineerings dienen die zum Einsatz kommenden Methoden im Wesentlichen zur systematischen Lösungsfindung differenzierterer Problemstellungen.

Zentraler Bestandteil eines Ganzheitlichen Produktionssystems ist der sog. Ordnungsrahmen (vgl. Kapitel 1.2). Ziel und Zweck des Ordnungsrahmens ist es, die unterschiedlichen Methoden mit den aus der Unternehmensstrategie hergeleiteten Gestaltungsprinzipien zu verknüpfen. Die Gestaltungsprinzipien orientieren sich dabei meist an den im Rahmen der oft zitierten IMVP-Studie (MIT) definierten fünf Kernprinzipien der schlanken Produktion: Wert, Wertstrom, Fluss, Pull, Perfektion [vgl. Wom90]. Aufgrund der unterschiedlichen Prozessgegebenheiten unterliegen die Prinzipien im direkten und indirekten Bereich unterschiedlichen Bedingungen. Auch hier gilt, dass die Abgrenzung aufgrund der angesprochenen Heterogenität nicht als trennscharf angesehen werden kann. Um die Bandbreite des Interpretationsspielraums darzustellen, wurde im Folgenden exemplarisch eine für den direkten Bereich typische standardisierte repetitive Tätigkeit einer für den indirekten Bereich charakteristi- 
schen kreativen Tätigkeit gegenübergestellt (vgl. Abbildung 4).

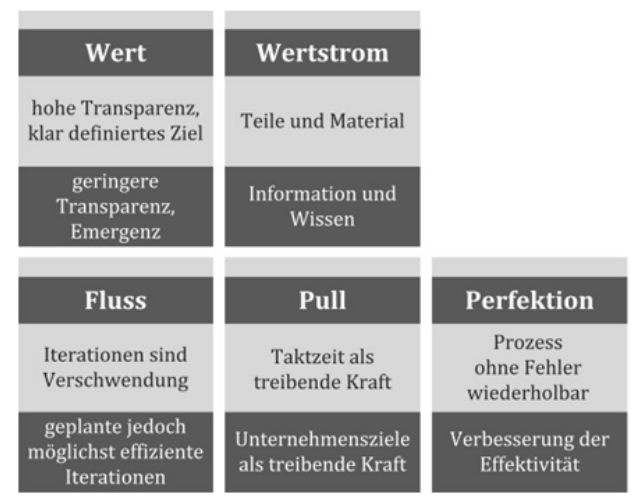

Abbildung 4. Gestaltungsprinzipien und Rahmenbedingungen (vgl. [Wom90; McM05])

Ist der Wert für den internen oder externen Kunden und damit das Ziel in einem stereotypischen direkten Prozess meist klar definiert und eindeutig nachvollziehbar, so ist bei einem indirekten Prozess in der Regel eine geringere Transparenz vorherrschend. Zudem zeichnen sich kreative Tätigkeiten häufig durch eine gewisse Emergenz aus, was die Planbarkeit erschwert. Der Wertstrom im direkten Bereich besteht in erster Linie aus Material sowie den zugehörigen Informationen. Im indirekten Bereich hingegen besteht der Wertstrom ausschließlich aus Informationen und Wissen. Im Sinne des Flusses sind Iterationen im direkten Bereich stets Verschwendung (z. B. Nacharbeit). Im indirekten Bereich hingegen können Iterationen bspw. in Planungs- oder Entwicklungsprozessen fester Bestandteil des Leistungsprozesses sein (z. B. Erzeugung von Varianten als Entscheidungsgrundlage).

Darüber hinaus werden in planenden und gestaltenden Bereichen bereits in der Aufbauorganisation Spannungsfelder implementiert, indem unterschiedliche Organisationseinheiten mit der Vertretung gegenläufiger Unternehmensinteressen betraut werden. Die so entstehenden Konflikte führen zwangsläufig zu Iterationen, tragen aber im Sinne der Unternehmensstrategie zu einer möglichst hohen Effektivität bei. Bei der Gestaltung ziehender Prozesse (Pull) im Bereich der Fließfertigung ist stets die Taktzeit die treibende Kraft. Im indirekten Bereich gelten die Unternehmensziele als Orientierung, wobei die im Prozess definierten Meilensteine als Taktgeber dienen. Ziel der Perfektion im direkten Bereich ist es den Prozess so zu gestalten, dass er möglichst effizient und ohne Fehler wiederholt werden kann. Perfektion im indirekten Bereich bedeutet hingegen insbesondere die Optimierung der Effektivität.

\section{2 ÜBERTRAgBarkeit DER PRINZIPIEN EINES BESTEHENDEN ORDNUNGSRAHMENS}

Auch wenn sich die Prinzipien bestehender Ordnungsrahmen historisch bedingt an den Gegebenheiten einer Fließfertigung orientieren und die dort vorherrschenden
Rahmenbedingungen teilweise diametral von denen im indirekten Bereich abweichen (vgl. Kap. 2.1), sind die hinter den Prinzipien stehenden Kernaussagen universell. Dies soll anhand des in Abbildung 5 dargestellten Ordnungsrahmens des Audi-Produktionssystems exemplarisch gezeigt werden.
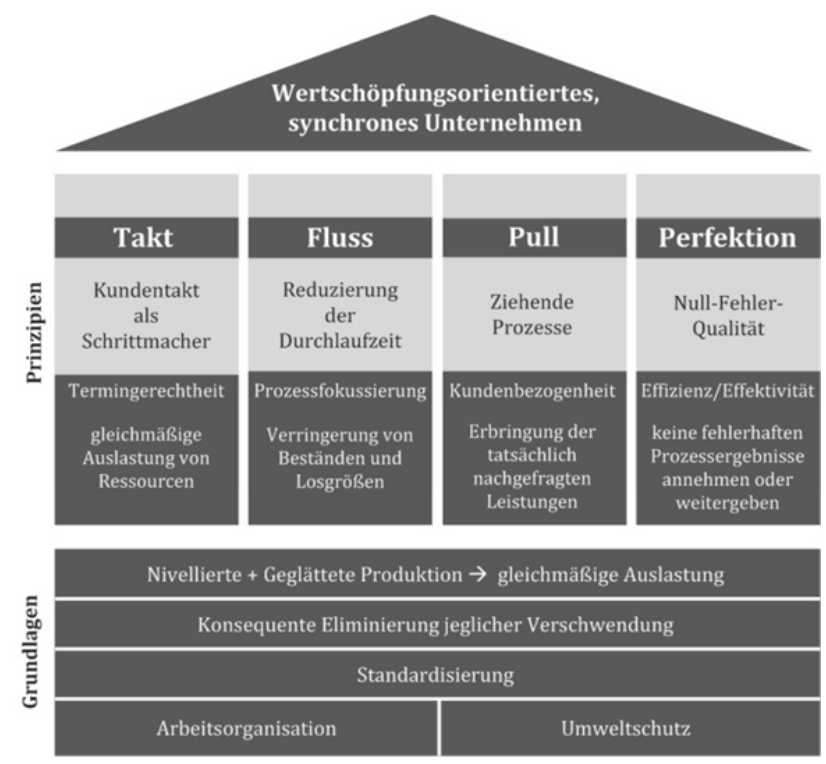

Abbildung 5. Audi-Produktionssystem [vgl. Fe11]

Im Audi-Produktionssystem wurden die Prinzipien Wert und Wertstrom [Wom90], wie auch beim wertschöpfungsorientierten Produktionssystem (BMW) oder Porsche-Produktionssystem durch das Prinzip Takt ersetzt. Auch wenn im indirekten Bereich offensichtlich kein Taktzwang besteht, gilt die hinter dem Prinzip stehende Forderung nach termingerechten Leistungen sowie gleichmäßig ausgelasteten Ressourcen in gleicher Weise. Unter Fluss im indirekten Bereich ist insbesondere die Fokussierung auf den Prozess zu verstehen. Dabei gilt es generiertes Wissen und Informationen zum geforderten Termin bereitzustellen und den Aufbau von Beständen so weit wie möglich zu vermeiden. Unter ziehenden Prozessen (Pull) im indirekten Bereich wird eine möglichst hohe Kundenbezogenheit verstanden, die durch das Erbringen der tatsächlich nachgefragten Leistung gewährleistet werden soll. Das Prinzip Perfektion gilt in sämtlichen Unternehmensbereichen gleichermaßen. Ziel ist es, keine fehlerhaften Prozessergebnisse zu erzeugen, anzunehmen oder weiterzugeben.

Beim Versuch der Einordnung der Methoden wird dennoch der ausgeprägte Fertigungsbezug offensichtlich. Gelingt es die Methoden des direkten Bereichs eindeutig einem der Prinzipien zuzuordnen, ist dies bei Methoden, die zur Lösung von Problemen in planenden und gestaltenden Bereichen eingesetzt werden, in der Regel nicht möglich. So kann beispielsweise die Methode des Pro- 
zessmappings $^{1}$ sämtlichen Prinzipien des dargestellten Ordnungsrahmens zugeordnet werden. Ein Prozessmapping kann dazu dienen, die Termintreue zu erhöhen (Takt), Bestände und Losgrößen zu minimieren (Fluss), die Kunden-/ Lieferantenbeziehungen durch klar definierte Schnittstellen zu verbessern (Pull) und die Effizienz respektive Effektivität (Perfektion) zu steigern. Dies zeigt, dass der bestehende, an den Rahmenbedingungen der Fertigung orientierte Ordnungsrahmen nur bedingt einer fortschreitenden Ausweitung des Einsatzgebietes Ganzheitlicher Produktionssysteme gerecht wird. So steht die verstärkte Integration indirekter Methoden einem nach wie vor an den Rahmenbedingungen der Fertigung ausgerichteten Ordnungsrahmen gegenüber.

\section{BEREICHSÜBERGREIFENDES ORDNUNGSMODELL}

Ziel eines bereichsübergreifenden Ordnungsmodells ist es, die Integrierbarkeit sämtlicher Unternehmensbereiche zu gewährleisten und eine zielgerichtete problemspezifische Methodenauswahl zu unterstützen. Dabei bleibt der traditionelle Ordnungsrahmen als Zielsystem bestehen und wird durch ein mehrdimensionales Ordnungssystem (Methodenwürfel) ergänzt. Die Trennung von Ziel- und Ordnungssystem bietet eine Reihe von Vorteilen. So können auftretende Problemstellungen anhand des Zielsystems einfach klassifiziert und in den Gesamtkontext des Unternehmens eingeordnet werden. Dabei sind die etablierten Grundprinzipien (Takt, Fluss, Pull, Perfektion) trotz der zunächst stark an der Fertigung orientierten Begrifflichkeiten bereichsunabhängig (vgl. Kap. 2.2). Durch die verstärkte Integration von Methoden im Zuge der Einbindung weiterer Unternehmensbereiche wird die Methodenauswahl anhand traditioneller Ordnungsrahmen jedoch zunehmend aufwendiger. Dies kann sich negativ auf die Umsetzung des Produktionssystems auswirken. Um dem entgegenzuwirken, wurde der im Folgenden vorgestellte Methodenwürfel konzipiert.

\subsection{METHODENWÜRFEL}

Der Methodenwürfel als bereichsübergreifendes Ordnungssystem verfolgt das Ziel, anhand von konkreten Problemstellungen geeignete Methoden zur Unterstützung einer zielgerichteten Lösungsfindung aufzuzeigen. Dazu werden die Methoden mit den Voraussetzungen ihrer Anwendung anhand der in Abbildung 6 dargestellten Ordnungskriterien verknüpft. Durch die Einordnung der Problemstellung nach Umfeld und Rahmenbedingungen, dem Adressat der Verbesserungsaktivität sowie dem Spezialisierungsgrad der gesuchten Methode, kann mit geringem Aufwand eine Vorauswahl getroffen werden. So ist es auch für weniger erfahrene Anwender möglich, die

\footnotetext{
${ }^{1}$ Das Prozessmapping dient zur Visualisierung von Ist-Prozessen, Aufzeigen von Potenzialen sowie zur systematischen Entwicklung eines verbesserten Sollprozesses.
}

Methoden eines Ganzheitlichen Produktionssystems gewinnbringend einzusetzen. Dies gilt insbesondere für kleine- und mittlere Unternehmen, die aus Kosten- und Kapazitätsgründen meist nicht in der Lage sind ein eigenes Ganzheitliches Produktionssystem zu etablieren.

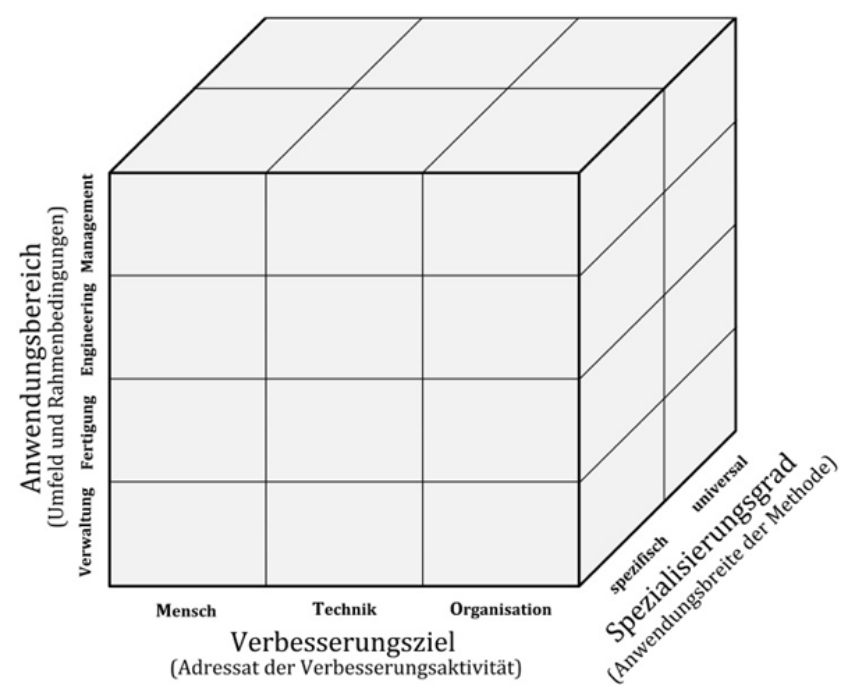

Abbildung 6. Methodenwürfel

Im Folgenden werden die Einordnungskriterien des Methodenwürfels im Einzelnen vorgestellt.

\subsubsection{ANWENDUNGSBEREICH (KRITERIUM 1)}

Die Anwendbarkeit von Methoden ist insbesondere von den Rahmenbedingungen der betrachteten Problemstellung abhängig. Um eine Klassifizierung der Methoden hinsichtlich ihrer Anwendungsvoraussetzungen durchführen zu können, ist eine sinnvolle Bereichseinteilung obligatorisch. Eine Unterscheidung, die lediglich zwischen direkten- und indirekten Unternehmensbereichen differenziert, ist aufgrund der in Kapitel 2.1 beschriebenen Heterogenität ebenso wenig zielführend wie eine zu feingliedrige Unterteilung. Im Rahmen des Methodenwürfels werden deshalb die Bereiche Management, Engineering, Fertigung und Verwaltung unterschieden. Dabei ist zu beachten, dass die Übergänge der Bereiche in der Praxis meist fließend sind. Zur Unterstützung einer gezielten Methodenauswahl ist es jedoch ausreichend eine Tendenz bezüglich des Umfeldes der betrachteten Problemstellung zu bestimmen.

Im Folgenden werden die sich in den Bereichen differenzierenden Rahmenbedingungen kurz erläutert: Aufgabe des Managements ist es, mögliche Entwicklungsrichtungen und Potenziale unter Einbeziehung vorhandener Ressourcen vorauszudenken und in einer zukunftsfähigen Strategie zu verankern. Entscheidungen sind hier aufgrund der hohen Dynamik und Komplexität der Um- 
weltbedingungen meist unter Ungewissheit ${ }^{2} \mathrm{zu}$ treffen. Aufgabe des Engineerings ist es, Lösungen für gegebene Problemstellungen zu erarbeiten. Dabei werden in einem kreativen Prozess sowohl vorhandene Ressourcen kombiniert als auch neue Ressourcen generiert. Der zu erbringende Prozessoutput ist meist Bestandteil eines übergeordneten Projekts. Hauptaufgabe dieser Bereiche ist es, die geforderten Ergebnisse termingerecht und in der geforderten Qualität zur Verfügung zu stellen (Effektivität). Bei verschiedenen Lösungsvarianten können den Handlungsalternativen meist konkrete Ergebnisse zugeordnet werden, die anhand der vom Management vorgegebenen Ziele bewertet werden können. Die auf diese Weise generierten Entscheidungen erfolgen nach entscheidungstheoretischen Gesichtspunkten unter Sicherheit. Im direkten Bereich der Fertigung existiert die höchste Planbarkeit. Hier gilt es in einem immer wiederkehrenden standardisierten Prozess vom Engineering vordefinierte Aufgaben, mit klar zugeordneten Ressourcen, abzuarbeiten. So ist die Qualität, Termintreue und Effizienz durch die hohe Prozessstandardisierung sowie die Abkopplung von Störgrößen weitestgehend vorbestimmt. Wie bereits in Kapitel 2.1 dargestellt, gelten in der Verwaltung, abgesehen von der Stofflichkeit des Leistungsergebnisses, ähnliche Rahmenbedingungen wie für die direkten Bereiche.

\subsubsection{VERBESSERUNGSZIEL (KRITERIUM 2)}

Mit dem Verbesserungsziel wird der primäre Adressat der betrachteten Verbesserungsaktivität bestimmt. Der Einsatz von Methoden eines Ganzheitlichen Produktionssystems dient dazu, die Arbeitsbedingungen der Mitarbeiter (Mensch), die im Unternehmen vorhandenen Maschinen, Anlagen und Systeme (Technik) und/ oder die Koordination von Personen, Sachmitteln und Informationen (Organisation) zu optimieren. Veränderungsprozesse in einem soziotechnischen System haben in der Regel Auswirkungen auf mehrere der o. g. Teilsysteme. Bei der Auswahl einer Methode ist jedoch ausschließlich der Adressat der Verbesserungsaktivität bzw. das primäre Verbesserungsziel von Interesse. Dabei ist darauf zu achten, dass das Teilsystem, an dem die Verbesserung vollzogen wird, nicht zwangsläufig auch der Adressat der Verbesserungsaktivität ist.

Ein Beispiel hierfür ist die Anwendung der Methode „Ergonomie“. Wird ein Manipulator eingesetzt, stellt dies zunächst ein Eingriff in das technische System dar. Der primäre Adressat der Verbesserungsaktivität ist jedoch der Mitarbeiter (Mensch), dessen Arbeitsbedingungen durch den Manipulatoreinsatz verbessert werden. Durch die Methode „Poka Yoke“ soll eine technische Lösung zur prospektiven Vermeidung menschlicher Fehler erarbeitet wer-

\footnotetext{
2 Bei Entscheidung unter Ungewissheit muss der Entscheider damit rechnen, dass unterschiedliche Umweltzustände eintreten können. So ist es nicht möglich Handlungsalternativen eindeutig Ergebnissen zuzuordnen [Bea05].
}

den. Primat der Anwendung ist die Vermeidung von Wiederholungsfehlern respektive stabile Prozesse (Organisation).

\subsubsection{SPEZIALISIERUNGSGRAD (KRITERIUM 3)}

Ziel dieses Ordnungskriteriums ist es, die Methoden hinsichtlich der Anwendungsbreite zu charakterisieren. Dabei geht es nicht um externe Rahmenbedingungen, wie sie bei der Zuordnung zu einem Anwendungsbereich ausschlaggebend sind (vgl. Kapitel 3.1.1). Stattdessen stehen die inhärenten Eigenschaften der Methode selbst im Vordergrund. Die Bandbreite der Methoden, die im Rahmen eines Ganzheitlichen Produktionssystems angewendet werden, reicht von Ansätzen zur Lösung spezifischer Problemstellungen bis hin zu übergeordneten nahezu universell anwendbaren Herangehensweisen zur Verbesserung von Prozessen mit differenzierten Eigenschaften. So ist die bereits unter 3.1.2 eingeführte Methode „Poka Yoke“ ausschließlich zur Vermeidung von Fehlern bei repetitiver Prozessausübung anzuwenden (spezifisch). Ein Ishikawa-Diagramm, welches zur systematischen Beschreibung von Problemen sowie zur Zuordnung möglicher Ursachen und Einflussgrößen genutzt wird, kann hingegen auf nahezu jede Art von Problemstellung angewandt werden (universell).

\subsection{EXEMPLARISCHE METHODENZUORDNUNG}

Um die hergeleiteten Kriterien hinsichtlich ihrer Praktikabilität zu bewerten, wurden exemplarisch die Methoden der VDI-Richtlinie 2870 (Ganzheitliche Produktionssysteme) in das konzipierte Ordnungssystem eingebunden. Bei der Zuordnung werden die Methoden mit mindestens einem Anwendungsbereich, einem Verbesserungsziel sowie genau einer Ausprägung des Spezialisierungsgrads verknüpft. So entsteht für jede Methode ein spezifischer Bereich, in dem eine zielführende Anwendung gewährleistet ist. Werden sämtliche dieser Bereiche übereinandergelegt, ergibt sich die folgende Darstellung (vgl. Abbildung 7).

Um eine gute Unterstützung bei der Methodenauswahl zu erreichen, ist es notwendig, dass sich die Eigenschaften der Methoden hinsichtlich der gewählten Kriterien möglichst heterogen darstellen. Die Abbildung zeigt, dass die Methoden des betrachteten Produktionssystems (VDI 2870) nahezu alle Bereiche des konzipierten Ordnungsrahmens abdecken und die gewählten Kriterien somit geeignet sind. Darüber hinaus gelingt es im Gegensatz zu bestehenden Ordnungsrahmen nun auch fertigungsfremde Methoden gleichrangig zuzuordnen. Die Ballung im Bereich Fertigung und Organisation ist zum einen auf die Ausrichtung des betrachteten Produktionssystems (VDI 2870) zurückzuführen, welches den Fokus klar auf die direkten Bereiche legt. Zum anderen ist es im direkten Bereich, aufgrund der sich wiederholenden, repetitiven Prozessgegebenheiten, möglich, eine Vielzahl problemspezifischer Methoden anzubieten. Hier ist es sinnvoll, 
den Ordnungsrahmen weiter zu untersetzen, indem bspw. die bekannten Kriterien Takt, Fluss, Pull und Perfektion integriert werden.

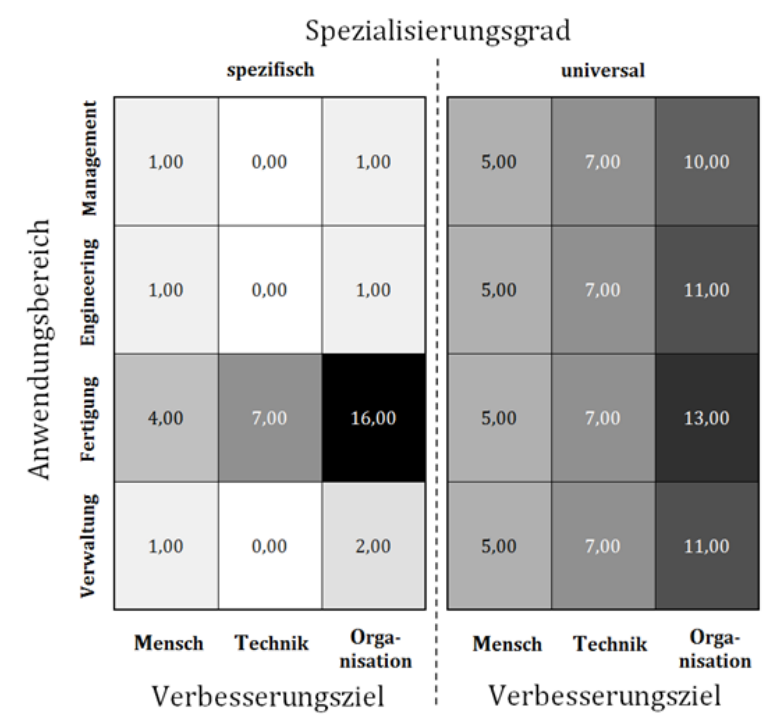

Abbildung 7. Zuordnung der Methoden der VDI 2870

Die weißen Flecken im Bereich Technik - Management/ Engineering/ Verwaltung sind auf eine untergeordnete Stellung der Technik in den genannten Bereichen zurückzuführen. Im Bereich der Verbesserungsziele Mensch und Organisation ist eine spezifische methodische Unterstützung hingegen anzustreben. Dies zeigt, dass es bei der konsequenten Ausweitung des Anwendungsgebietes eines, an den Gegebenheiten der Fertigung ausgerichteten Managementsystems nicht ausreicht, lediglich die bestehenden Methoden an die Rahmenbedingungen der indirekten Bereiche anzupassen. Vielmehr sind es die im Rahmen einer Erweiterung häufig integrierten Methoden, wie Problemlösung oder Prozessmapping, mit denen die besten Ergebnisse zu erzielen sind.

\section{ZUSAMMENFASSUNG UND AUSBLICK}

Der vorliegende Artikel thematisiert die Auswirkungen einer verstärkten Integration indirekter Unternehmensbereiche in das traditionell fertigungsseitig geprägte Managementkonzept Ganzheitlicher Produktionssysteme. Dazu werden zunächst die Grundlagen Ganzheitlicher Produktionssysteme vermittelt sowie die Rahmenbedingungen für eine Anwendung im indirekten Bereich analysiert und vom direkten Bereich abgegrenzt. Die sich insbesondere in den Bereichen des Engineerings und Managements stark von der Fertigung differenzierenden Rahmenbedingungen begründen den anhaltenden Trend zur Integration weiterer Methoden. Die wachsende Methodenzahl steht einem nach wie vor häufig stark an den Bedingungen der Fertigung ausgerichteten Ordnungsrahmen gegenüber. Es wird dargestellt, dass die fünf Kernprinzipien der schlanken Produktion auch für die indirekten Bereiche von Bedeutung sind - zur gezielten problem-spezi- fischen Methodenauswahl es jedoch notwendig ist, ein gesondertes, alle Unternehmensbereiche abdeckendes Ordnungssystem zu etablieren. Hierzu wurde das Konzept einer übergeordneten mehrdimensionalen Ordnungssystematik vorgestellt und die Kriterien durch die Zuordnung erster Methoden getestet. Ziel der weiteren Arbeit wird es sein, gezielt weitere Methoden zu integrieren sowie die gewählten Ordnungskriterien so zu verfeinern, dass eine problemspezifische Methodenauswahl nahezu automatisiert erfolgen kann.

\section{LITERATUR}

[Bau02] Baust, Hans; Bergmeier, Barbara: Ganzheitliche Produktionssysteme. Gestaltungsprinzipien und deren Verknüpfung. 1. Aufl., Köln: Wirtschaftsverlag Bachem, 2002, ISBN 978-3-87284072-1

[Bea05]

Bea, Franz X.; Friedl, Birgit; Schweitzer, Marcell: Allgemeine Betriebswirtschaftslehre - Bd. 2: Führung. 10. Aufl., Stuttgart: UTB, 2005, ISBN 38252-1082-0

[Fau09] Faust, Peter: Zweite Lean-Welle - Die sieben Thesen. In: ZWF Zeitschrift für wirtschaftlichen Fabrikbetrieb 104, Nr. 3, 2009, S. 157-163

[Fe11] Fehr, Sebastian; Sauber, Kay.; Bader, Helge; Schmidt, Thorsten; Völker, Michael: Integration eines Ganzheitlichen Produktionssystems im indirekten Bereich - Besonderheiten in der Umsetzung bei der AUDI AG. In: ZWF Zeitschrift für wirtschaftlichen Fabrikbetrieb 106, Nr. 10, 2011, S. 711715

[Keß09] Keßler, Stephan et al., 2009. Ganzheitliche Produktionssysteme für Logistikdienstleister. https://eldorado.tudortmund.de/handle/2003/26441, Abgerufen am 18.07.2012

[Korg04] Korge, Axel; Scholtz, Oliver: Ganzheitliche Produktionssysteme - Produzierende Unternehmen innovativ organisieren und führen. In: wt Werkstatttechnik online , 94, 2004, 1/2, S. 2-6.

[Kra03] Kramer, Oliver: Methode zur Optimierung der Wertschöpfungskette mittelständi scher Betriebe. München: Herbert Utz Verlag, 2003, ISBN 3-83160211-5 
[Lay08] Lay, Gunter; Zanker, Cristoph: Ganzheitliche Produktionssysteme auch in kleinen und mittleren Unternehmen. In: Industrial Engineering - Fachzeitschrift des REFA-Bundesverbandes 61, 2008, 2, S. $16-20$

[Lay08a] Lay, Gunter: Von Modernisierungsinseln zu integrierten Produktionssystemen: Ein Leitfaden für die strategieorientierte Verknüpfung betrieblicher Modernisierungsmaßnahmen in kleinen und mittleren Unternehmen, 1.Aufl., Frankfurt: VDMA Verlag, 2008, ISBN 978-3-8163-0551-4

[McM05] McManus, Hugh L., Allen Haggerty, and Earll Murman: http://lean.mit.edu/ component/docman/doc_details/775lean-engineering-doing-the-right-thingright?Itemid=332, Abgerufen am 25.03.2012] The Aeronautical Journal, Vol. 111, No. 116, February 2007, pp. 105-114. (Originally published in slightly different form in Proceedings of the 1st International Conference on Innovation and Integration in Aerospace Sciences, Queen's University Belfast, Northern Ireland, UK, August 2005.)

[Mil00] Milberg, Joachim; Reinhardt, Gunther (Hrsg.): Unternehmenspolitik im Wandel. In: Münchner Kolloquium 2000: ... nur der Wandel bleibt - Wege jenseits der Flexibilität. München: Herbert Utz Verlag, 2000. S. 311-331, ISBN 978389675-923-8

[Mos09] Moser, Patrick: Stakeholdermanagement zur optimalen Gestaltung strategischen Wandels. Hamburg: Diplomica Verlag, 2009, ISBN 3-8366-5218-8

[Ohn93] Ohno, Taiichi: Das Toyota Produktionssystem. 1. Aufl., Frankfurt: Campus Verlag, 1993, ISBN 3-593-34946-9

[Port99] Porter, Michael E.: Wettbewerbsvorteile (Competitive Advantage): Spitzenleistungen erreichen und behaupten. Frankfurt: Campus Verlag, 1999, ISBN 3-5933-6178-7

[Spa03] Spath, Dieter: Ganzheitlich produzieren: Innovative Organisation und Führung. 1. Aufl., Stuttgart: LOG_X Verlag, 2003, ISBN 3-9322-9822-5
[Spa04] Spath, Dieter: Weltmarktführer bei der Arbeitsorganisation - Was müssen wir noch tun?, In: Kongress Arbeit und Organisation 2004. Mehr Produktivität durch Systeme, Methoden und Standards; Standortsicherung durch Interessenausgleich und Zusammenarbeit; Berlin, 2004

[Spe00] Specht, Dieter; Hellmich, Kai: Management der Zulieferbeziehungen in dynamischen Produktionsnetzen. In: Wildemann, Horst (Hrsg.): Supply Chain Management. München: TCW Transfer-Centrum Verlag, 2000, S. 89115

[Töpf07] Töpfer, Armin: Betriebswirtschaftslehre: Anwendungs- und prozessorientierte Grundlagen. Berlin: Springer Verlag, 2007, ISBN 978-3-540-49394-5

[VDI2870] Verein Deutscher Ingenieure (VDI): Ganzheitliche Produktionssysteme Grundlagen, Einführung und Bewertung (Lean production systems - Basic principles, introduction, and review). Blatt 1, Berlin: Beuth Verlag, 2012

[Wit06] Wittenstein, Anna-Katharina; Wesoly, Michael; Moeller, Georg; Schneider, Ralph: Lean Office 2006. http://www.schlankeprozesse.de/data/St udie_Lean_Office-Highlights.pdf, Abgerufen am 25.03.2012

[Wom90] Womack, James P.; Jones, Daniel T.; Roos, Daniel: The Machine that changed the World: The Story of Lean Production. New York: Harper Collins, 1990, ISBN 978-0-060-97417-6 